\title{
Do Graduates from Arts-Related Disciplines have a Higher Impact on Unemployment than Graduates from the Science-Related Disciplines?
}

\author{
Victoria Kakooza ${ }^{1}$, Robert Wamala ${ }^{2}$, James Wokadala ${ }^{3} \&$ Thomas Bwire ${ }^{4}$ \\ ${ }^{1} \mathrm{PhD}$ Student, School of Statistics, College of Business and Management Science, Makerere University. Kampala, \\ Uganda \\ ${ }^{2}$ Deputy Director, Directorate of Research and Graduate Training, Makerere University,Kampala, Uganda \\ ${ }^{3}$ Lecturer- School of Statistics, College of Business and Management Science, Makerere University.Kampala, \\ Uganda \\ ${ }^{4}$ Economic Reaserch Function,Bank of Uganda, Uganda \\ Correspondence: Victoria Kakooza, PhD Student, School of Statistics, College of Business and Management Science, \\ Makerere University. Kampala, Uganda. E-mail: victoriakakooza@gmail.com
}

Received: June 2, 2019

doi:10.5430/ijhe.v8n4p52
Accepted: June 29, 2019

Online Published: July 2, 2019

\begin{abstract}
There have been several attempts in developing countries to reduce both graduate and overall unemployment; with the majority attempts centered on changes in the education sector. To better understand this avenue, this study intends to comparatively establish the impact of the two broad discipline categories of- Arts and science related disciplines- on the overall unemployment. The study employed the Vector autoregressive (VAR) model to analyse Uganda's data between 1991 and 2017. The findings of the study showed that the arts/humanities graduates have a slightly higher impact on unemployment than their counterparts from the science/technology disciplines in the short run in Uganda; with both groups of graduates having no significant effect on unemployment in the long run.
\end{abstract}

Keywords: graduates, arts-related disciplines, science-related disciplines, unemployment, vector error correction model

\section{Introduction}

Employment today has taken the norm of "survival-for-the-fittest'. The reduction of unemployment today has been threatened by technology acceleration that has bombarded the labour markets in an unexpected way (Peters, Jandric, $\&$ Means, 2019). This technology acceleration has arrived promising employers of services without wages, leaves, complaints, and all human errors (Arthur, 2019). Slowly but surely, the issue of unemployment is becoming a reality. Unemployment not only threatens the future work of graduates (Peters, Jandric, \& Means, 2019) but threaten the future of the higher education sector. As a bell ringing, there needs to be an urgent prioritization of tackling unemployment, like never before, starting with various studies researching the different aspects and angles of unemployment. In addition, some scholars have argued that education- skills mismatch drives graduates to entrepreneurship, (Ganco, \& Raffiee, 2018) hence reducing unemployment; while others say that skills mismatch negatively impacts entering into entrepreneurship (Stenard, \& Sauermann, 2016). This brings to light the required skills in different job markets- warranting research in different countries.

Literature on the link between unemployment and higher education is still inconclusive. Some studies have evidenced that Higher education reduces unemployment (Nunez \& Livanos, 2010 in Europe; Lee \& chung, 2015; and Wasim, Raza, \& Sharif, 2017 in Pakistan); while others have found that education increased unemployment (Erdem \& Tugcu, 2012 in Turkey; Li, Whalley, \& Xing, 2014 in China. Others like Snieska et al. (2015) found no significant effect of education on employment among selected countries of the European Union (EU). These evidences however, have predominantly been cited in developed countries, with limited studies in the developing countries. As the relationship between education and unemployment still a paradox, studies on the extent to which academic disciplines impact overall unemployment is rare. Academic discipline has been regarded as having a substantial impact on a person's employment (Altonji, Kahn, \& Speer, 2014; Nunez \& Livanos, 2010). Evidences from literature of developed countries seem to favour graduates from the science-related disciplines (Nunez \& Livanos, 2010). Computer science degree for instance, has been considered as a golden ticket to employment (Chow, 
2019). This leaves one wondering about the unemployment impact of the other disciplines.

In the past, Uganda has had more students enrolled in the arts related disciplines, than in the science related disciplines. Flipping the coin, the trend of unemployment over the years has fluctuated, instead of reducing. Given that oversupply of graduates is considered a major cause of unemployment (Nel \& Neale-Shutte, 2013); could it be that the graduates from the arts related disciplines have contributed more to this persistent unemployment problem than those from the science related disciplines? Besides, the arts related disciplines include social work, social administration, literature, history, linguistics, arts, social sciences, law, philosophy, music, performing arts, and archaeology; Mass communication, and Religious Studies. These graduates have a wider spectrum of jobs compared to those from science/ technology. A big percentage of them are employed in the informal sector, public service and other private institutions. With the wide range of employers and a big informal sector employing $91.4 \%$ of youths (UBOS, 2018), giving the aforementioned graduates an employment advantage, one would think otherwise. On the other hand, graduates of Science/technology disciplines including medicine, pharmacy, engineering, computer science, and general sciences; require more of the subject knowledge at their jobs, making them more specialized and prone to unemployment. This makes the comparative impact of these two groups of graduates on unemployment not an obvious matter.

The objective of the paper therefore is to add to the existing literature the disaggregated and comparative impact of the two broad disciplines of the higher education sector, on overall unemployment in a case of a developing country such as Uganda. In this revolution of technology, do graduates from the arts related disciplines cause more unemployment than those from the science related disciplines? The remaining part of the paper is structured into 5 sections: literature review, methodology, results, discussion and policy recommendations, as well as the Conclusion.

\section{Literature Review}

Graduates from the Arts related disciplines are known for their excellent communication, interpersonal skills, and their ability to present effective debates and negotiations (Lyonette, Hunt, \& Baldauf, 2017). As much as these skills do shape good leaders and are a basis for international relations and are thus expected to act as a catalyst for employment, their employability has been questioned by studies that found otherwise. Abel, Deitz, \& Su, (2014) did a study on the employability of study majors of college graduates and found that social sciences and arts had higher probabilities of being unemployed than those from engineering. Another study by Altbeker \& Storme (2013) found that commerce and some science graduates in South Africa and had higher unemployment rates than others. Spaull (2013) mentioned that engineering and accounting graduates had lower chances of unemployment in South Africa. A study by Mncayi, \& Dunga, (2016) found that arts graduates, in particular, human resources, politics, and industrial psychology among others, took a longer time to get employment in South Africa. In favour of the science related cources, Ryan, (2012) evidenced lower unemployment of engineering and science graduates; Aden, (2017) noted that dentistry, medicine, and veterinary degrees had lower rates of unemployment in Canada. Woya, (2019) found over $80 \%$ of statistics graduates employable. Research by Woya, (2019) and Livanos, (2010) found that graduates of computer science and polytechnics had more employment opportunities than graduates of humanities and social sciences in Greece. In Uganda, Walter, et al.2017) reported that $84 \%$ Bachelor of Agriculture (BAG) degree graduates were employed immediately after graduation

It should be noted however that, previous studies on the impact of academic disciplines on unemployment; do not give a unified conclusion. Leech-Wilkinson, (2013) found that social science graduates had lower unemployment rates; Pauw, Oosthuizen, \& Van Der Westhuizen, (2008) found that graduates from social science and humanities had lower unemployment rates in South Africa, than their counterparts in sciences, computer studies, and mathematics. These findings are inconclusive but insightful concerning the relationship between study discipline and unemployment. However, it should be noted that these studies looked at the employability of the students/ graduates but not their impact on overall unemployment rate in the country; leaving a research gap. Whether the employability of different disciplines is translated into less overall unemployment in the country, remains a subject of research. This forms the basis of this study. Another concern noted in the majority of these studies is that the research focused on single academic subjects( Nunez \& Livanos,2010), which may not provide a wider perspective for macroeconomic policy making. These studies tend to guide students, parents and donors on which study disciplines to offer or financially support. This study therefore adds to the existing literature the impact of the two major academic disciplines on overall unemployment.

Oversupply of graduates has been named as one of the major reasons cited for higher unemployment rates (Gokulsing, \& Tandrayen, 2018) Researchers have also cited job- skills mismatches as another cause of unemployment resulting from graduates of different study fields (Shierholz, Davis, \& Kimball, 2014; Verhaest, 
Sellami, \& Van der Velden, 2017). This can be explained by the fact that job- skills mismatches lead several graduates into finding occupations that are not aligned to their field of study thereby causing unemployment of workers suitable for those posts. This implies that both the science and arts graduates are liable to negatively impact unemployment. The disciplines are subject to more mismatches plus their effect on general unemployment; would differ with each labour market.

\section{Methodology}

The current study utilized data from numerous sources: Annual data on unemployment, Foreign Direct Investment; Gross capital Formation (GCF); and Gross Domestic product (GDP); were obtained from the World Bank data base. Data on Arts/ humanities graduates and Science/ technology graduates were obtained from a purposive sample of three public universities in Uganda. This annual data from 1991 to 2017 was later quarterised using the software inbuilt in ms-excel computer package.

\subsection{Model Specification}

Vector autoregressive (VAR) model is popular for regressing multivariate time series that are not co-integrated together but have the same order of integration individually. The simultaneous equations have values of all the variables dependent on the lagged values of all of them, plus the error term. Here all variables tend to be endogenous. The Vector Autoregressive (VAR) model was chosen for analysis, knowing that most macroeconomic variables are non stationary at level. This model was based on a multivariate model that determines unemployment; taking into consideration both the demand and supply factors. Unemployment results from a distortion in the labour market, where supply of labour exceeds the demand for it . The standardized VAR model is stated in equation 1.

$$
y_{t}=A_{1} y_{t-1}+A_{2} y_{t-2}+\cdots+A_{p} y_{t-p}+B x_{t}+\mu_{t}
$$

Where $\mathrm{t}=1,2, \ldots, \mathrm{T} ; y_{t}$ is a $1 \mathrm{xk}$ matrix of time series dimension; $y_{t} \sim \mathrm{I}(1)$ and each $y_{t}$ is influenced by $x_{t}$ independent time series

Tailoring equation 1 to suit the current study, the VAR system took the form in equations 2 to 7

$$
\begin{aligned}
& U^{N} E M P_{t}=A_{1} A R T S_{t-1}+A_{2} S C I_{t-2}+A_{2} G D P_{t-2}+A_{2} G C F_{t-2}+A_{p} F D I_{t-p}+\mu_{t} \\
& \text { ARTS }_{t}=A_{1} A R T S_{t-1}+A_{2} S C I_{t-2}+A_{2} G D P_{t-2}+A_{2} G C F_{t-2}+A_{p} F D I_{t-p}+\mu_{t} \\
& S C I_{t}=A_{1} A R T S_{t-1}+A_{2} S C I_{t-2}+A_{2} G D P_{t-2}+A_{2} G C F_{t-2}+A_{p} F D I_{t-p}+\mu_{t} \\
& G D P_{t}=A_{1} A R T S_{t-1}+A_{2} S C I_{t-2}+A_{2} G D P_{t-2}+A_{2} G C F_{t-2}+A_{p} F D I_{t-p}+\mu_{t} \\
& G C F_{t}=A_{1} A R T S_{t-1}+A_{2} S C I_{t-2}+A_{2} G D P_{t-2}+A_{2} G C F_{t-2}+A_{p} F D I_{t-p}+\mu_{t} \\
& \text { FDI }_{t}=A_{1} A R T S_{t-1}+A_{2} S C I_{t-2}+A_{2} G D P_{t-2}+A_{2} G C F_{t-2}+A_{p} F D I_{t-p}+\mu_{t}
\end{aligned}
$$

Where UNEMP denotes unemployment, (Arts graduates) Arts/ humanities graduates, Sci graduates (Science/ technology graduate); Gross Domestic product (GDP), Gross capital Formation (GCF); and FDI Foreign Direct Investment.

\section{Results}

\subsection{Test for Unit Root}

The Augmented Dickey-Fuller-test (ADF) was used to test the each endogenous variable for the presence of unit roots and integration. For unit roots to be absent, the following models have to be satisfied. The ADF unit root results are presented in Table 1.

Table 1. Unit Root Test Results

\begin{tabular}{llcll}
\hline Variables & Level & \multicolumn{3}{l}{ First Difference } \\
\cline { 2 - 5 } & Value & P-value & Value & P-value \\
\hline UNEMPLOYMENT & -1.834313 & 0.3622 & -15.16652 & 0.0000 \\
Arts graduates & -1.642302 & 0.4575 & -13.96825 & 0.0000 \\
Science graduates & -1.235173 & 0.6570 & -10.12639 & 0.0000 \\
GDP_GROWTH & -1.628523 & 0.4645 & -12.93956 & 0.0000 \\
FDI_M_DOLLARS & -1.412729 & 0.5735 & -14.62538 & 0.0000
\end{tabular}

Note: Estimate were obtained using the Augmented Dickey Fuller Tests. UNEMPLYT denotes unemployment, Arts 
graduates ( Arts/ humanities graduates), Sci graduates (Sciencel technology graduate); Gross Domestic product $(G D P)$, Gross capital Formation (GCF); FDI_M_DOLLARS denotes Foreign Direct Investment in million US dollars.

Table 1 presented the presence of stationarity for all variables at first difference, since in all scenarios, the ADF statistics were greater than the corresponding critical values, the null hypothesis was not rejected at $10 \%, 5 \%$, and $1 \%$ significance levels.

\subsection{Optimal Lag Selection}

Another vital step was to determine the lag length that is optimal in the VECM analysis.

The Akaike information criterion (AIC), Schwarz information criterion (SIC), LR test statistic (LR), and Hannan-Quinn information criterion (HQ) test statistics criteria was used for lag selection., the results are shown in Table 2.

Table 2. Results of the Optimal Lag Selection

\begin{tabular}{|c|c|c|c|c|c|}
\hline Model & Lag & LR & AIC & SIC & HQ \\
\hline \multirow{3}{*}{$\begin{array}{l}\text { UNEMPLYT =f(ARTS SCI GCF, } \\
\text { GDP_GROWTH, FDI_M_DOLLARS, }\end{array}$} & 0 & NA & 2.719768 & 2.963544 & 2.787381 \\
\hline & 1 & 35.61338 & 0.925380 & 1.217910 & 1.006516 \\
\hline & 2 & $9.537078 *$ & $0.475543 *$ & $0.816828 *$ & $0.570201 *$ \\
\hline
\end{tabular}

Note. Sequential modified LR test statistic (LR), Akaike information criterion (AIC), Schwarz information criterion (SIC), and Hannan-Quinn information criterion (HQ). UNEMPLYT denotes unemployment, Arts graduates ( Arts/ humanities graduates), Sci graduates (Science/ technology graduate); Gross Domestic product (GDP), Gross capital Formation (GCF); FDI_M_DOLLARS denotes Foreign Direct Investment in million US dollars.

Lag (2) was the best choice given by the criteria used, as shown in Table 2. This was then used in the proceeding analyses.

\subsection{Co-Integration of Variables}

The Johansen Test was used to test for the presence of co-integration among the variables. This test is based on the Trace and Maximum Eigen test statistics The co-integration of variables was tested basing on the null hypothesis that all the variables are co-integrated against the alternative that the variables are not co-integrated. The null hypothesis cannot be rejected when the p-value is greater than $5 \%$, in both tests. The results are presented in Table 3 .

Table 3. Results for the Co-integration Test

\begin{tabular}{llllllll}
\hline \multirow{2}{*}{ Regression Model } & \multicolumn{2}{l}{ Data Trend: } & None & None & Linear & Linear & Quadratic \\
\cline { 2 - 8 } & Test Type & $\begin{array}{l}\text { No } \\
\text { Intercept }\end{array}$ & Intercept & Intercept & Intercept & Intercept \\
\cline { 3 - 8 } & & No Trend & No Trend & No Trend & Trend & Trend \\
\hline $\begin{array}{l}\text { UNEMPLYT } \\
\text { SCI GCF, GDP_GROWTH, } \\
\text { FDI_M_DOLLARS,) }\end{array}$ & Trace & 1 & 0 & 0 & 0 & 1 \\
\hline
\end{tabular}

Note: UNEMPLYT denotes unemployment, Arts graduates (Arts/ humanities graduates), Sci graduates (Science/ technology graduate); Gross Domestic product (GDP), Gross capital Formation (GCF); FDI_M_DOLLARS denotes Foreign Direct Investment in million US dollars.

Johansen test results are shown in Table 2, and they present the absence of co-integration among the variables. The Trace and Maximum Eigen value tests reveal that there is no co-integrating equation in the model system. As such, the variables do not have a long run relationship.

4.4 Estimation Results for the VAR Analysis

Following the fact that the variables were not co-integrated, the VAR model was used to analyse the results as shown in Table 4. 
Table 4. VAR Regression Results

\begin{tabular}{|c|c|c|c|c|c|c|}
\hline & UNEMPLYT & $\mathrm{GCF}$ & $\begin{array}{l}\text { GDP_GRO } \\
\mathrm{H}\end{array}$ & $\begin{array}{l}\text { FDI_M_DOLL } \\
\text { ARS }\end{array}$ & ARTS & $\mathrm{SCI}$ \\
\hline UNEMPLYT(-1) & $\begin{array}{l}1.222860 * * * \\
(0.16376) \\
{[7.46749]}\end{array}$ & $\begin{array}{l}-881.0898 * * * \\
(223.345) \\
{[-3.94497]}\end{array}$ & $\begin{array}{r}-1.623310 \\
(1.22227) \\
{[-1.32811]}\end{array}$ & $\begin{array}{l}-251.3694 * * * \\
(53.2340) \\
{[-4.72198]}\end{array}$ & $\begin{array}{r}-1307.630 \\
(1139.90) \\
{[-1.14714]}\end{array}$ & $\begin{array}{r}109.6604 \\
(442.786) \\
{[0.24766]}\end{array}$ \\
\hline UNEMPLYT(-2) & $\begin{array}{c}-0.436881 * * * \\
(0.15128) \\
{[-2.88782]}\end{array}$ & $\begin{array}{l}861.9775 * * * \\
(206.333) \\
{[4.17761]}\end{array}$ & $\begin{array}{c}0.444704 \\
(1.12917) \\
{[0.39383]}\end{array}$ & $\begin{array}{l}237.1878 * * * \\
(49.1791) \\
{[4.82294]}\end{array}$ & $\begin{array}{l}2237.948 * * \\
(1053.07) \\
{[2.12516]}\end{array}$ & $\begin{array}{c}219.6933 \\
(409.059) \\
{[0.53707]}\end{array}$ \\
\hline $\operatorname{GCF}(-1)$ & $\begin{array}{c}-7.39 \mathrm{E}-05 \\
(0.00025) \\
{[-0.29700]}\end{array}$ & $\begin{array}{c}0.389871 \\
(0.33948) \\
{[1.14845]}\end{array}$ & $\begin{array}{c}0.001666 \\
(0.00186) \\
{[0.89654]}\end{array}$ & $\begin{array}{r}-0.054235 \\
(0.08091) \\
{[-0.67029]}\end{array}$ & $\begin{array}{c}-1.152601 \\
(1.73260) \\
{[-0.66524]}\end{array}$ & $\begin{array}{l}1.768226 \text { ** } \\
(0.67302) \\
{[2.62732]}\end{array}$ \\
\hline $\operatorname{GCF}(-2)$ & $\begin{array}{c}5.92 \mathrm{E}-05 \\
(0.00021) \\
{[0.27880]}\end{array}$ & $\begin{array}{c}-0.585623 * * \\
(0.28949) \\
{[-2.02293]}\end{array}$ & $\begin{array}{c}-0.002978 * \\
(0.00158) \\
{[-1.87975]}\end{array}$ & $\begin{array}{r}-0.054417 \\
(0.06900) \\
{[-0.78865]}\end{array}$ & $\begin{array}{c}0.585079 \\
(1.47750) \\
{[0.39599]}\end{array}$ & $\begin{array}{l}-1.154457 * * \\
(0.57392) \\
{[-2.01151]}\end{array}$ \\
\hline GDP_GROWTH(-1) & $\begin{array}{r}-0.021209 \\
(0.03423) \\
{[-0.61953]}\end{array}$ & $\begin{array}{c}24.06866 \\
(46.6896) \\
{[0.51550]}\end{array}$ & $\begin{array}{c}0.227065 \\
(0.25551) \\
{[0.88867]}\end{array}$ & $\begin{array}{c}10.04888 \\
(11.1284) \\
{[0.90299]}\end{array}$ & $\begin{array}{c}273.5713 \\
(238.293) \\
{[1.14805]}\end{array}$ & $\begin{array}{c}33.02678 \\
(92.5631) \\
{[0.35680]}\end{array}$ \\
\hline GDP_GROWTH(-2) & $\begin{array}{r}-0.014939 \\
(0.03017) \\
{[-0.49516]}\end{array}$ & $\begin{array}{c}32.97456 \\
(41.1493) \\
{[0.80134]}\end{array}$ & $\begin{array}{r}-0.310849 \\
(0.22519) \\
{[-1.38037]}\end{array}$ & $\begin{array}{r}9.083594 \\
(9.80788) \\
{[0.92615]}\end{array}$ & $\begin{array}{r}-217.4425 \\
(210.017) \\
{[-1.03536]}\end{array}$ & $\begin{array}{r}-123.1086 \\
(81.5794) \\
{[-1.50906]}\end{array}$ \\
\hline FDI_M_DOLLARS(-1) & $\begin{array}{r}-0.000463 \\
(0.00061) \\
{[-0.75758]}\end{array}$ & $\begin{array}{c}1.444898 * \\
(0.83381) \\
{[1.73288]}\end{array}$ & $\begin{array}{r}-0.003470 \\
(0.00456) \\
{[-0.76050]}\end{array}$ & $\begin{array}{l}0.780856 * * * \\
(0.19874) \\
{[3.92908]}\end{array}$ & $\begin{array}{c}-4.207972 \\
(4.25559) \\
{[-0.98881]}\end{array}$ & $\begin{array}{l}-4.182020 * * \\
(1.65305) \\
{[-2.52988]}\end{array}$ \\
\hline FDI_M_DOLLARS(-2) & $\begin{array}{r}0.000981 \\
(0.00083) \\
{[1.18754]}\end{array}$ & $\begin{array}{l}3.881508 * * \\
(1.12700) \\
{[3.44412]}\end{array}$ & $\begin{array}{c}0.005487 \\
(0.00617) \\
{[0.88970]}\end{array}$ & $\begin{array}{c}0.183236 \\
(0.26862) \\
{[0.68214]}\end{array}$ & $\begin{array}{l}12.85878 * * \\
(5.75192) \\
{[2.23556]}\end{array}$ & $\begin{array}{l}4.681432 * * \\
(2.23429) \\
{[2.09527]}\end{array}$ \\
\hline ARTS(-1) & $\begin{array}{c}-8.65 \mathrm{E}-05^{*} \\
(5.1 \mathrm{E}-05) \\
{[-1.68166]}\end{array}$ & $\begin{array}{c}-0.188174 * * \\
(0.07018) \\
{[-2.68147]}\end{array}$ & $\begin{array}{c}-0.000114 \\
(0.00038) \\
{[-0.29601]}\end{array}$ & $\begin{array}{c}-0.052863 * * * \\
(0.01673) \\
{[-3.16047]}\end{array}$ & $\begin{array}{l}0.820752 * * \\
(0.35816) \\
{[2.29158]}\end{array}$ & $\begin{array}{r}0.031886 \\
(0.13912) \\
{[0.22919]}\end{array}$ \\
\hline ARTS(-2) & $\begin{array}{l}0.000199 * * * \\
(6.6 \mathrm{E}-05) \\
{[3.04233]}\end{array}$ & $\begin{array}{r}-0.002599 \\
(0.08938) \\
{[-0.02908]}\end{array}$ & $\begin{array}{c}0.000513 \\
(0.00049) \\
{[1.04805]}\end{array}$ & $\begin{array}{l}0.064739 * * * \\
(0.02130) \\
{[3.03884]}\end{array}$ & $\begin{array}{r}-0.004800 \\
(0.45618) \\
{[-0.01052]}\end{array}$ & $\begin{array}{c}0.183349 \\
(0.17720) \\
{[1.03470]}\end{array}$ \\
\hline $\mathrm{SCI}(-1)$ & $\begin{array}{l}7.63 \mathrm{E}-05 \\
(0.00010)\end{array}$ & $\begin{array}{l}0.689723 * * * \\
(0.13825)\end{array}$ & $\begin{array}{l}0.000664 \\
(0.00076)\end{array}$ & $\begin{array}{l}0.154764 * * * \\
(0.03295)\end{array}$ & $\begin{array}{l}0.171607 \\
(0.70558)\end{array}$ & $\begin{array}{l}0.682548 * * \\
(0.27408)\end{array}$ \\
\hline
\end{tabular}




\begin{tabular}{lcccccc}
\hline & {$[0.75315]$} & {$[4.98906]$} & {$[0.87734]$} & {$[4.69679]$} & {$[0.24321]$} & {$[2.49034]$} \\
SCI(-2) & & & & & & \\
& $-0.000365^{* * *}$ & -0.134970 & -0.000843 & $-0.102082 * * *$ & -0.979880 & $-0.859718 * * *$ \\
& $(9.9 \mathrm{E}-05)$ & $(0.13448)$ & $(0.00074)$ & $(0.03205)$ & $(0.68633)$ & $(0.26660)$ \\
& {$[-3.70505]$} & {$[-1.00367]$} & {$[-1.14575]$} & {$[-3.18488]$} & {$[-1.42770]$} & {$[-3.22473]$} \\
C & & & & & & \\
& $0.901128 *$ & 106.9229 & $10.34311 * * *$ & -12.76461 & -170.8160 & 434.2362 \\
& $(0.34490)$ & $(470.398)$ & $(2.57429)$ & $(112.119)$ & $(2400.80)$ & $(932.573)$ \\
& {$[2.61274]$} & {$[0.22730]$} & {$[4.01785]$} & {$[-0.11385]$} & {$[-0.07115]$} & {$[0.46563]$} \\
\hline R-squared & & & & & & \\
Adj. R-squared & 0.974378 & 0.994062 & 0.748513 & 0.985429 & 0.958626 & 0.982641 \\
Sum sq. resids & 0.948755 & 0.988124 & 0.497027 & 0.970858 & 0.917252 & 0.965281 \\
S.E. equation & 0.403736 & 751012.3 & 22.49210 & 42664.94 & 19562705 & 2951767. \\
F-statistic & 0.183425 & 250.1687 & 1.369066 & 59.62727 & 1276.803 & 495.9643 \\
Log likelihood & 38.02841 & 167.4023 & 2.976356 & 67.63051 & 23.16986 & 56.60576 \\
Akaike AIC & 16.09990 & -164.3522 & -34.15207 & -128.5017 & -205.1017 & -181.4614 \\
Schwarz SC & -0.247992 & 14.18818 & 3.772166 & 11.32013 & 17.44814 & 15.55692 \\
Mean dependent & 0.385824 & 14.82199 & 4.405981 & 11.95395 & 18.08195 & 16.19073 \\
S.D. dependent & 2.621385 & 3059.752 & 6.536811 & 485.2935 & 7703.990 & 3905.930 \\
\hline
\end{tabular}

Determinant resid covariance (dof adj.)

4. $32 \mathrm{E}+16$

Determinant resid covariance

$5.28 \mathrm{E}+14$

Log likelihood

$-636.5864$

Akaike information criterion

57.16691

Schwarz criterion

60.96980

\begin{abstract}
Note: Figures denote the coefficients, standard errors(), and t-statistics[]. $\Delta$ denotes first difference,-1 denotes lag 1,-2 denotes lag 2.*,** and *** means significant at $10 \%, 5 \%$ and $1 \%$ respectively. UNEMPLYT denotes unemployment, Arts graduates ( Arts/ humanities graduates), Sci graduates (Sciencel technology graduate); Gross Domestic product (GDP), Gross capital Formation (GCF); FDI_M_DOLLARS denotes Foreign Direct Investment in million US dollars.
\end{abstract}

From the VAR analysis, the corresponding model system is given in equations

UNEMPLYT $=\mathrm{C}(1) * \mathrm{UNEMPLYT}(-1)+\mathrm{C}(2) * \mathrm{UNEMPLYT}(-2)+\mathrm{C}(3) * \mathrm{GCF}(-1)+\mathrm{C}(4) * \mathrm{GCF}(-2)+\mathrm{C}(5) * \mathrm{GDP}$ GROWTH(-1) + C $(6) *$ GDP_GROWTH(-2) + C(7)*FDI_M_DOLLARS $(-1)+\mathrm{C}(8) *$ FDI_M_DOLLARS $(-2)+\mathrm{C}(9) *$ ARTS $(-1)+\mathrm{C}(10) *$ ARTS $(-2)$ $+\mathrm{C}(11) * \mathrm{SCI}(-1)+\mathrm{C}(12) * \mathrm{SCI}(-2)+\mathrm{C}(13)$

$\mathrm{GCF}=\mathrm{C}(14) * \mathrm{UNEMPLYT}(-1)+\mathrm{C}(15) * \mathrm{UNEMPLYT}(-2)+\mathrm{C}(16) * \mathrm{GCF}(-1)+\mathrm{C}(17) * \mathrm{GCF}(-2)+\mathrm{C}(18) * \mathrm{GDP} \_\mathrm{GROWTH}(-1)+$ $\mathrm{C}(19) *$ GDP_GROWTH(-2) + C(20)*FDI_M_DOLLARS(-1) + C(21)*FDI_M_DOLLARS(-2) + C(22)*ARTS(-1) + $\mathrm{C}(23) * \operatorname{ARTS}(-2)+\mathrm{C}(24) * \mathrm{SCI}(-1)+\mathrm{C}(25) * \mathrm{SCI}(-2)+\mathrm{C}(26)$

GDP_GROWTH $=\mathrm{C}(27) * \mathrm{UNEMPLYT}(-1)+\mathrm{C}(28) * \mathrm{UNEMPLYT}(-2)+\mathrm{C}(29) * \mathrm{GCF}(-1)+\mathrm{C}(30) * \mathrm{GCF}(-2)+$ C $(31) *$ GDP_GROWTH(-1) + C(32)*GDP_GROWTH(-2) + C(33)*FDI_M_DOLLARS(-1) + C(34)*FDI_M_DOLLARS(-2) + $\mathrm{C}(35) * \operatorname{ARTS}(-1)+\mathrm{C}(36) * \operatorname{ARTS}(-2)+\mathrm{C}(37) * \mathrm{SCI}(-1)+\mathrm{C}(38) * \mathrm{SCI}(-2)+\mathrm{C}(39)$

FDI_M_DOLLARS $=\mathrm{C}(40) * \mathrm{UNEMPLYT}(-1)+\mathrm{C}(41) * \operatorname{UNEMPLYT}(-2)+\mathrm{C}(42) * \mathrm{GCF}(-1)+\mathrm{C}(43) * \mathrm{GCF}(-2)+$ C(44)*GDP_GROWTH(-1) + C(45)*GDP_GROWTH(-2) + C(46)*FDI_M_DOLLARS(-1) + C(47)*FDI_M_DOLLARS(-2) + $\mathrm{C}(48) * \operatorname{ARTS}(-1)+\mathrm{C}(49) * \operatorname{ARTS}(-2)+\mathrm{C}(50) * \mathrm{SCI}(-1)+\mathrm{C}(51) * \mathrm{SCI}(-2)+\mathrm{C}(52)$

ARTS $=\mathrm{C}(53) * \mathrm{UNEMPLYT}(-1)+\mathrm{C}(54) * \mathrm{UNEMPLYT}(-2)+\mathrm{C}(55) * \mathrm{GCF}(-1)+\mathrm{C}(56) * \mathrm{GCF}(-2)+\mathrm{C}(57) * \mathrm{GDP} \_\mathrm{GROWTH}(-1)+$ C(58)*GDP_GROWTH(-2) + C(59)*FDI_M_DOLLARS(-1) + C(60)*FDI_M_DOLLARS(-2) + C(61)*ARTS(-1) + $\mathrm{C}(62) * \operatorname{ARTS}(-2)+\mathrm{C}(63) * \mathrm{SCI}(-1)+\mathrm{C}(64) * \mathrm{SCI}(-2)+\mathrm{C}(65)$

$\mathrm{SCI}=\mathrm{C}(66) * \mathrm{UNEMPLYT}(-1)+\mathrm{C}(67) * \mathrm{UNEMPLYT}(-2)+\mathrm{C}(68) * \mathrm{GCF}(-1)+\mathrm{C}(69) * \mathrm{GCF}(-2)+\mathrm{C}(70) * \mathrm{GDP} \_\mathrm{GROWTH}(-1)+$ 
$\mathrm{C}(71) * \mathrm{GDP}$ GROWTH(-2) + C(72)*FDI_M_DOLLARS(-1) + C(73)*FDI_M_DOLLARS(-2) + C(74)*ARTS(-1) + $\mathrm{C}(75) * \operatorname{ARTS}(-2)+\mathrm{C}(76) * \mathrm{SCI}(-1)+\mathrm{C}(77) * \mathrm{SCI}(-2)+\mathrm{C}(78)$

The Wald test also conducted to affirm the short run causality between the variables of interest. The results are in Table 5.

Table 5. Results for the Wald test

\begin{tabular}{|c|c|c|c|}
\hline Null Hypothesis & Probability & Conclusion & \\
\hline $\mathrm{C}(9)=\mathrm{C}(10)=0$ & 0.0036 & \multirow{2}{*}{$\begin{array}{l}\text { Bi-directional } \\
\text { graduates }\end{array}$} & unemployment and Arts \\
\hline$C(53)=C(54)=0$ & 0.0417 & & \\
\hline $\mathrm{C}(11)=\mathrm{C}(12)=0$ & 0.0009 & Unidirectional & graduates \\
\hline$C(66)=C(67)=0$ & 0.3227 & & \\
\hline
\end{tabular}

Note: $\mathrm{C}(9), \ldots, \mathrm{C}(67)$ are coefficients

Focusing on the major variables under study, there is a short term impact of graduates of both disciplines on unemployment, as affirmed by the Wald test results. From the regression analysis, in Table.4, a small impact from both graduate disciplines to unemployment is shown. The impact is though significant. The results exhibit that the arts graduates (Science graduates) reduce (increase) unemployment immediately in the first lag, but the vice-versa is true after the second lag. Wald test continue to affirm the bi-directional relationship between unemployment and Arts graduates, compared to a unidirectional relationship from Science graduates to unemployment.

\section{Discussion and Policy Recommendations}

This study disaggregated higher education graduates by two academic discipline groupings in order to establish the link between higher education and unemployment. In the findings, the study reveals that the two broad academic discipline categories had only a short term impact on the overall unemployment of Uganda. This finding is contrary to those of Mirica (2014) who did not find the impact of education on unemployment in the short run. However, the findings validate the argument that higher education causes unemployment for developing countries (Erdem \& Tugcu, 2012; Li, Whalley, \& Xing, 2014). From this study, stating such a notion needs one to integrate the concept of time frame.

Although researchers have agreed on the possibility of mass unemployment accruing from technological advancement (Peters, Jandric, \& Means, 2019); there is still hope for this to be reversed. Short term policies should be employed so as to reduce unemployment in Uganda, or other similar developing countries; with a guide that the arts graduates immediately increase unemployment as compared to the science and technology graduates. Such policies include redesigning the both disciplines, especially the arts related disciplines of the higher education institutions, so as to incorporate more entrepreneurship knowledge and training. The students' minds need to be unlocked with creativity skills, that unveils the unique potential exhibited in the different disciplines. As much as the science/ technology disciplines are good, the arts/ humanities disciplines continue to play unique roles of eloquent orators, theoretical and historical founded analysis, as well as philosophers; skills which cannot be easily substituted by graduates from the science disciplines. They surely have their place in the labour markets, which should be taken on gladly. Such differentiation brought about by varying fields of study could lead to irreplaceable workers and life-long knowledge that could prepare students for the future uncertainties (Király, \& Géring, 2019). Furthermore, there is need for employers-higher education collaborations, aimed at better allocating and aligning graduates from the two academic disciplines, to their fields of work. This could help reduce unemployment as partnerships of this nature have been proved to exhibit positive returns on employability of graduates, in addition to their skills (Dimopoulos, 2018).

More to this finding, there was a bi-directional relationship between unemployment and graduates from the Arts related courses established that demonstrates the existence of a reverse causality. The evidence of a reverse causal relationship has not been established by prior studies (e.g Nunez \& Livanos, 2010); mainly because of the findings were obtained using methodologies like the logit model- that cannot decompose the bi-causality impact of the variables under study. A unidirectional causality running from graduates from the Science related disciplines to unemployment was also established. This implies that more unemployment results into a higher demand for higher education only from the Arts related disciplines. 


\section{Conclusion}

In Conclusion, the study gives an developing country's perspective and sheds light on the comparative bi- causal impact of graduates from the arts related and science related disciplines on unemployment in Uganda. The study reveals that the arts/humanities graduates have a slightly higher impact on unemployment than their counterparts from the science/technology disciplines in the short run in Uganda. Both discipline graduates do not have a long run effect on unemployment. This study contributes to the literature the differing effects in the two time periods. The study also adds that overall unemployment significantly affects the growth of higher education graduates from the arts disciplines. Further studies need to establish the reasons why this is so.

\section{Acknowledgement}

Acknowledgement is made to the Makerere university- SIDA funding and the Swedish government, which supported the accomplishment of this study.

\section{References}

Abel, J. R., Deitz, R., \& Su, Y. (2014). Are recent college graduates finding good jobs?. Current Issues in Economics and Finance, 20(1).

Aden, I. (2017). Impact of Education on Unemployment Evidence From Canada.

Altbeker, A., \& Storme, E. (2013). Graduate unemployment in South Africa: A much exaggerated problem. Centre for Development and Enterprise.

Altonji, J, G., Kahn, L. B., \& Speer, J. D. (2014). Cashier or Consultant? Entry Labor Market Conditions, Field of Study, and Career Success NBER Working Paper No. 20531. https://doi.org/10.3386/w20531

Armitage, D., Bhabha, H., Dench, E., Hamburger, J., Hamilton, J., Kelly, S., ... \& Simpson, J. (2013). The teaching of the arts and humanities at Harvard College: Mapping the future. Cambridge, ma, Harvard University, 31.

Arthur, C. (2019). Is Entrepreneurial Education the Solution to the Automation Revolution?. In Education and Technological Unemployment (pp. 79-93). Springer, Singapore. https://doi.org/10.1007/978-981-13-6225-5_6

Breznitz, S. M., \& Zhang, Q. (2019). Determinants of graduates' entrepreneurial activity. Small Business Economics, 1-18. https://doi.org/10.1007/s11187-019-00171-8

Cameron,J. (2019). The value of liberal arts education for finding professional employment. https://doi.org/10.1108/JARHE-07-2018-0141

Chow, T.Y. (2019). Is a Computer Science Degree the Golden Ticket? Effects of Race, Place, and Degree Institution on First Job Outcomes in Texas. University of California, Santa Barbara, ProQuest Dissertations Publishing. 13805520.

Devi, V. N., Vijayalata, Y., Raju, N. V., Murthy, J. N., Praveen, J., \& Raju, K. V. S. (2019). Significance of Digital Technologies in Teaching and Learning: A Case Study on Finishing School, Griet. Y. and Raju, NV Ganapathi and Murthy, Jandhyala N. and Praveen, J. and Raju, KVS, Significance of Digital Technologies in Teaching and Learning: A Case Study on Finishing School, Griet (April 24, 2019). https://doi.org/10.2139/ssrn.3377600

Dimopoulos, A. (2018). 'From studies to employment'.

Erdem, E., \& Tugcu, C. T. (2012). Higher Education and Unemployment: a cointegration and causality analysis of the case of Turkey. European Journal of Education, 47(2), 299- 309.

https://doi.org/10.1111/j.1465-3435.2012.01526.x

Ganco, M., \& Raffiee, J. (2018). Job-Education Match and Immigrant Entrepreneurship: Theory and Evidence from Graduates in Science and Engineering. Working Paper.

Gokulsing, D \& Tandrayen, V. (2018). Globalisation, Higher Education and Youth Unemployment: The Case of Mauritius. Conference Paper, $5^{\text {th }}$ International Conference on Managing Organizations In Africa, Mauritius. .

Higgs, J., Cork, S., \& Horsfall, D. (Eds.). (2019). Challenging future practice possibilities. BRILL. https://doi.org/10.1163/9789004400795

Hill, C. B., \& Pisacreta, E. D. ( 2019). The Economic Benefits and Costs of a Liberal Arts Education. The Andrew W. Mellon Foundation. https://creativecommons.org/licenses/by-nc/4.0/.

Johansen, S. (1995). Likelihood-Based Inference in Cointegrated Vector Auto Regressive Models. Advanced Texts in 
Econometrics, Oxford University Press Inc, NewYork. https://doi.org/10.1093/0198774508.001.0001

Juselius, K. (2006). The cointegrated VAR model: methodology and applications. Oxford university press.

Király, G., \& Géring, Z. (2019). Introduction to 'Futures of Higher Education'special issue. Futures.

Lee, K. W., \& Chung, M. (2015). Enhancing the link between higher education and employment. International Journal of Educational Development, 40, 19-27. https://doi.org/10.1016/j.ijedudev.2014.11.014

Leech-Wilkinson, R. (2013). What Do Social Science Graduates Do? The Campaign for Social Science report. The Higher Education Statistics Agency Publishers.

Li, S., Whalley, J., \& Xing, C. (2014). China Economic Review China's higher education expansion and unemployment of college graduates. China Economic Review, 30(71103019), 567-582. https://doi.org/10.1016/j.chieco.2013.08.002

Livanos, I. (2010). The relationship between higher education and labour market in Greece: the weakest link? Higher Education, 60(5), 473-489. https://doi.org/10.1007/s10734-010-9310-1

Lyonette, C., Hunt, H., \& Baldauf, B. (2017). Occupations and skills of Arts, Humanities and Social Sciences Graduates and Postgraduates. A report prepared for The British Academy. Warwick Institute for Employment Research University of Warwick.

Mirica, A. (2014). Higher Education -A Solution to Unemployment ? Case Study. Romanian Statistical Review, (3), 63-75.

Mncayi, P., \& Dunga, S. H. (2016). Career choice and unemployment length: A study of graduates from a South African university. Industry and Higher Education, 30(6), 413-423. https://doi.org/10.1177/0950422216670500

Nunez, I., \& Livanos, I. (2010). Higher education and unemployment in Europe: an analysis of the academic subject and national effects. Higher Education, 59(4), 475-487. https://doi.org/10.1007/s10734-009-9260-7

Pauw, K., Oosthuizen, M., \& Van Der Westhuizen, C. (2008). Graduate Unemployment In The Face Of Skills Shortages: A Labour Market Paradox 1. South African journal of economics, 76(1), 45-57. https://doi.org/10.1111/j.1813-6982.2008.00152.x

Peters, M. A., Jandrić, P., \& Means, A. J. (2019). Introduction: Technological Unemployment and the Future of Work. In Education and Technological Unemployment (pp. 1-12). Springer, Singapore. https://doi.org/10.1007/978-981-13-6225-5_1

Ryan, C. (2012). Field of Degree and Earnings by Selected Employment Characteristics: 2011. American Community Survey Briefs. ACSBR/11-10. US Census Bureau.

Shierholz, H., Davis, A., \& Kimball, W. (2014). The class of 2014: The weak economy is idling too many young graduates. Economic Policy Institute, 1.

Spaull, N. (2013). South Africa's education crisis: The quality of education in South Africa 1994-2011. Johannesburg: Centre for Development and Enterprise, 1-65.

Stenard, B. S., \& Sauermann, H. (2016). Educational mismatch, work outcomes, and entry into entrepreneurship. Organization Science, 27(4), 801-824. https://doi.org/10.1287/orsc.2016.1071

Uganda Bureau of Statistics (UBOS). (2018). Statistical Abstract 2018. Kampala, Uganda.

Vandenbussche, J., Aghion, P., \& Meghir, C. (2006). Growth, distance to frontier and composition of human capital. Journal of economic growth, 11(2), 97-127. https://doi.org/10.1007/s10887-006-9002-y

Verhaest, D., Sellami, S., \& Van der Velden, R. (2017). Differences in horizontal and vertical mismatches across countries and fields of study. International Labour Review, 156(1), 1-23. https://doi.org/10.1111/j.1564-913X.2015.00031.x

Walter, O., Stephen, W. K., Enos, K. K., Elly, N., \& Duncan, O. (2017). Responsiveness of agricultural training curricula in African universities to labour market needs: the case of Gulu University in Uganda. African Journal of Rural Development (AFJRD), 2(1978-2017-1974).

World Bank. (2018). World Bank Open data - World Bank Group.

Woya, A. A. (2019). Employability among Statistics Graduates: Graduates' Attributes, Competence, and Quality of Education. Education Research International, 2019. https://doi.org/10.1155/2019/7285491 